\title{
Electromechanical model of RF MEMS switches
}

\author{
L. X. Zhang, Y.-P. Zhao
}

\begin{abstract}
With the recent rapid growth of Radio Frequency Micro-Electro-Mechanical Systems (RF MEMS) switches, there has developed an emergent requirement for more accurate theoretical models to predict their electromechanical behaviors. Many parameters exist in the analysis of the behavior of the switch, and it is inconvenient for further study. In this paper, an improved model is introduced, considering simultaneously axial stress, residual stress, and fringing-field effect of the fixed-fixed bridge structure. To avoid any unnecessary repetitive model tests and numerical simulation for RF MEMS switches, some dimensionless numbers are derived by making governing equation dimensionless. The electromechanical behavior of the fixed-fixed bridge structure of RF MEMS switches is totally determined by these dimensionless numbers.
\end{abstract}

\section{1}

\section{Introduction}

In recent years, Micro-Electro-Mechanical Systems (MEMS) technology has grown rapidly and entered into many communication and defense applications [1-3]. At present, as the development in MEMS technology [4], Radio Frequency (RF) MEMS is one of the fastest growing areas in commercial MEMS technology. As a novel switch, RF MEMS switches have a myriad application in radar system and wireless communications $[5,6]$. Comparing to semiconductor switches widely used in millimeter wave integrated circuits and microwave circuits, the novel device has a low insertion loss, good isolation, low return loss, high frequency, good Q-factor, and a low cost and power consumption.

RF MEMS switches consist of a thin metal membrane, entitled the "bridge", suspended over a center conductor, and one beam fixed on the ground conductor. There are

Received: 4 February 2002/Accepted: 19 May 2002

\section{X. Zhang, Y.-P. Zhao ( $₫)$}

State Key Laboratory of Nonlinear Mechanics,

Institute of Mechanics, Chinese Academy of Sciences,

Beijing 100080, PR China

E-mail: yzhao@lnm.imech.ac.cn (Zhao YP)

The supports from the Key Project from the Chinese Academy of Sciences (No. KJCX2-SW-L2), projects from the NSFC (Nos 19928205, No. 50131160739 and No. 10072068), and the National "973" Project (Grant No. G1999033103) are gratefully acknowledged. The authors would like to thanks Prof. Yilong Hao of Institute of Microelectronics, Peking University, for helpful discussion. mainly three types of beams: cantilever, fixed-fixed beam, and torsion beam $[7,8]$, and fixed-fixed beam are under extensive research at present. The present paper goes on study of RF MEMS switches based on simple fixed-fixed beam.

Theoretical model can offer proper and convenient approach for numerical calculations, and promote the design of devices. At present, some theories and models of RF MEMS switches have been established gradually based on conventional theories. Because of miniaturization and electromechanical coupling, some new phenomena and factors, such as axial stress due to beam large deflection, residual stress in thin films and so on, become comparatively more important. To design and optimize RF MEMS switches further, it is essential to set up more accurate models.

The dimensionless numbers are useful for scaling purposes and for organizing experimental model tests and numerical calculations to avoid any unnecessary repetition of the results in dimensionless space $[9,10]$. In the analysis of improved models of RF switches, taking more physical quantities into account, the dimensionless numbers can play an important role in making the analysis more convenient.

In the present paper, a brief review of the existing models is presented in Sect. 3, then an improved model is put forward in Sect. 4 by considering axial stress caused by beam large deflection, residual stress as well as fringingfield effect, and four dimensionless numbers are obtained by making governing equation dimensionless. Some existing models of RF switches and data of pull-in voltage are reformulated using the dimensionless numbers and analyzed further.

The following begins with brief introduction on structure and working principle of RF MEMS switches.

\section{2}

\section{Structure and working principle of RF MEMS switches}

The schematic view of RF MEMS switches as analyzed in this paper is shown in Fig. 1. The device consists of transmission line, hinge, movable plate formed by using electroplated $\mathrm{Au}$ or $\mathrm{Cu}$, and substrate. One SEM photo of the switch is shown in Fig. 2.

The switch can be fabricated using surface micromachining techniques, electroplating techniques and dry releasing technique compatible to the (millimeter wave integrated circuit) MMIC fabrication processes. A brief fabrication sequence for the RF MEMS switches described in Fig. 1 is shown in Fig. 3 [11]. A ground plate and a 


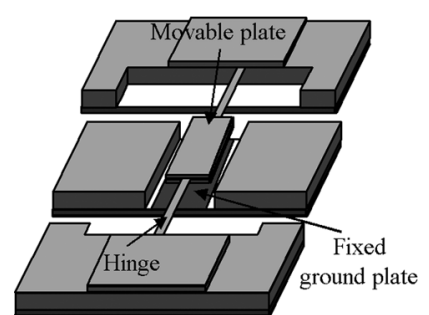

Fig. 1. Schematic of one kind of RF MEMS switches

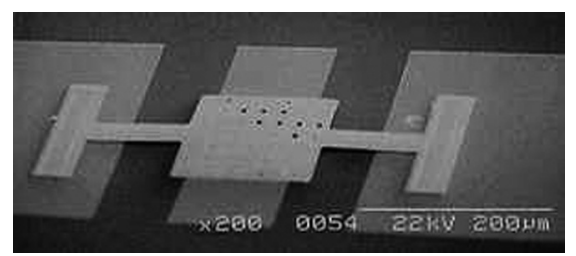

Fig. 2. SEM of a fixed-fixed RF MEMS switch

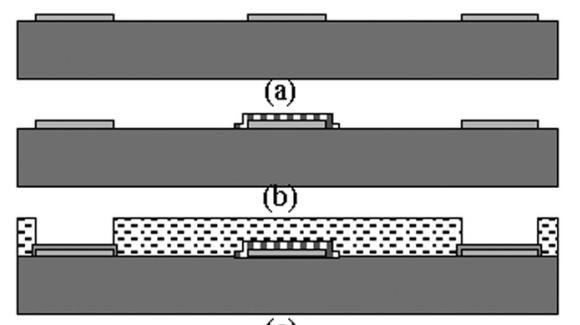

(c)

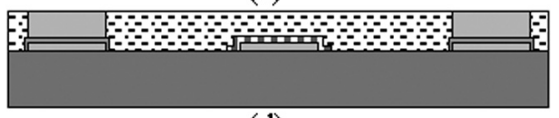

(d)

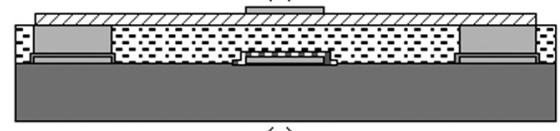

(e)

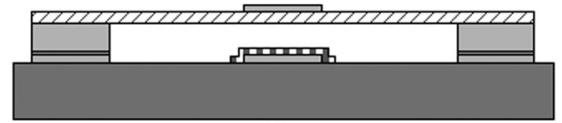

(f)

Fig. 3. Fabrication sequences of RF MEMS switches shown in Fig. 1

transmission line were formed on substrate (shown in Fig. 3(a)). High dielectric layer was deposited on top of the formed transmission line (shown in Fig. 3(b)). A patterned seed layer was formed for electroplating the metal posts, and a photoresist was deposited on the ground plate and the dielectric layer (shown in Fig. 3(c)). Metal posts formed on seed layer (shown in Fig. 3(d)). A hinge and a movable plate were formed, and a mass was deposited on the top of the formed plate (shown in Fig. 3(e)). The movable plate and hinge were released by etching the sacrificial layer (photoresist) (shown in Fig. 3(f)).

In theoretical analysis, the parameters of RF MEMS switches involve materials constants (such as elastic modulus, Poisson's ratio and so on), geometrical dimensions of the fixed-fixed beam moment of inertia of the cross-section, the gap between the movable and the fixed ground plates, and electrostatics. The analysis of these

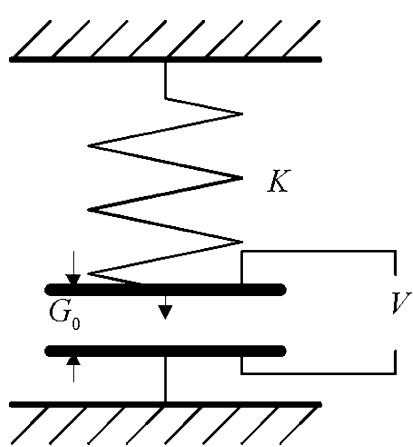

Fig. 4. 1D lumped model for pull-in test

parameters can be simplified to great extent by the following analysis in this paper.

The essential of the problem of the RF MEMS switches is the coupling of the mechanics and the electrostatics, which governs the behavior of the structure of RF MEMS switches [12]. The device controls mechanically electrical current or signal, by using the on/off impedance ratio. While a voltage is applied between the movable and the fixed ground plates of the switch as shown in Fig. 1, the movable plate subjected to the electrostatic force moves down onto the fixed ground plate. When the pull-in voltage is reached, the switch is in the down state, or the blocking state, when no voltage is applied it is in the up state, or pass-though state [13].

3

\section{Existing models}

Electrostatic pull-in is a famous instable behavior of an elastic supported structure with regard to parallel-plate electrostatic actuation [14]. Despite the pull-in event is sudden and sharp, the actuation voltage can be accurately measured at wafer level by the standard electrical test equipment with a microscope. Existing models have no sufficient accuracy, so that there has developed an emergent requirement for more accurate model to approach the design of RF MEMS switches.

\section{1}

\section{D lumped model [14-16]}

The 1D lumped model approximates pull-in structure by a single rigid parallel-plate capacitor suspended above a fixed ground plane by an ideal linear spring as shown in Fig. 4. The model is the simplest and most intuitive analytically, but its accuracy is very poor. Its purpose is for first-cut analysis to gain physical insight, explore design options and understand overall behavior.

The pull-in voltage $V_{\mathrm{pi}}$ can be expressed by the following equation

$V_{\mathrm{pi}}=\sqrt{\frac{8 K_{\mathrm{eff}} G_{0}^{3}}{27 \varepsilon_{0} A}}$,

where $\varepsilon_{0}$ is the vacuum permittivity, $K_{\text {eff }}$ the effective spring constant, $G_{0}$ and $A$ the initial gap and the overlapping area between the movable and the fixed plates, respectively. 


\section{2}

\section{D distributed model [15-19]}

Compared with the 1D lumped model, the 2D distributed model can contain the influences of the fringing field, residual stress, axial stress, and so on. Thus it can attain more accurate solutions. The 2D distributed model generally includes the cantilever (shown in Fig. 5) and fixedfixed beam (shown in Fig. 6) structures.

The governing equation of the beam as shown in Fig. 5 or Fig. 6 is

$\tilde{E} I \frac{\partial^{4} w}{\partial x^{4}}=\frac{\varepsilon_{0} V^{2} B}{2\left(G_{0}-w\right)^{2}}$,

where $\tilde{E}, \widehat{I}, w, B$ are the effective modulus, the effective moment of inertia of the cross-section, the deflection and the width of the beam, respectively. $V$ is the voltage applied between the movable and the ground plates on the fixed substrate. Following assumptions have been supposed to simplify the analysis:

(i) residual stress in the fixed-fixed beam is ignored.

(ii) error derived from nonhomogeneous distribution of charge, after the movable fixed-fixed beam deflects, is ignored.

(iii) fringing field effect is ignored.

(iv) small deflection is assumed.

In theoretical modeling, some models considering residual stress are established [15], which are represented as the following governing equation

$\tilde{E} I \frac{\partial^{4} w}{\partial x^{4}}-T_{\mathrm{r}} \frac{\partial^{2} w}{\partial x^{2}}=\frac{\varepsilon_{0} V^{2} B}{2\left(G_{0}-w\right)^{2}}$,

where $T_{\mathrm{r}}$ is the residual force of fixed-fixed beam.

Some models involving residual stress and fringing field are analyzed $[15,16]$, which derived the governing equation as follows

$\tilde{E} \bar{I} \frac{\partial^{4} w}{\partial x^{4}}-T_{\mathrm{r}} \frac{\partial^{2} w}{\partial x^{2}}=\frac{\varepsilon_{0} V^{2} B}{2\left(G_{0}-w\right)^{2}}\left(1+f_{\mathrm{f}}\right)$,

where $f_{\mathrm{f}}$ is the fringing field correction. The residual stress and fringing field effect are interpreted further in detail in Sect. 4.1.

\section{4}

\section{Model improvement}

Because of the miniaturization and special fabrication technology, it is necessary to take some the effects such as residual stress, fringing field effect and axial stress into account simultaneously. Some research about residual stress and fringing field effect is referred in Sect. 3.2 in modeling. In this section, in addition to further interpretation of residual stress and the fringing effect, another important factor axial stress, is analyzed. Then an improved model is established, involving these important factors.

The detailed schematic of fixed-fixed bridge structure is shown in Fig. 7, where $w$ is the deflection of the fixed-fixed beam, $B, t$ and $L$ and are the width, the thickness, and the initial length of the fixed-fixed beam, respectively.

\section{1 \\ Residual stress and fringing field effect}

\section{(i) Residual stress}

Residual stress, due to the mismatch of both thermal expansion coefficient and crystal lattice period between substrate and thin film, is unavoidable in surface micromachining techniques, so that accurate and reliable data of residual stress is crucial to the proper design of the MEMS devices concerned with the techniques $[20,21]$. Therefore the residual stress is an attractive research topic in the development of the Microsystem Technology (MST). Considering the fabrication sequence of RF MEMS switches in Fig. 3, the residual stress is very important and inevitable to the device. Residual force can be expressed as

$T_{\mathrm{r}}=\hat{\sigma} B t$

where $\hat{\sigma}$ is the residual stress, equal zero for cantilever, and $\hat{\sigma}=\sigma_{0}(1-v)$ for fixed-fixed beam, where $\sigma_{0}$ is the biaxial residual stress [15].

\section{(ii) Fringing field effect}

A uniform magnetic field cannot drop abruptly to zero at an edge as shown in Fig. 8(a). In actual situation, there is always a "fringing field" existing, and a more realistic situation including "fringing field" modification is illustrated in Fig. 8(b).

If fringing field effect is taken into account, the first order fringing-field correction $[15,16]$ is denoted as

$f_{\mathrm{f}}=0.65 \frac{G_{0}-w}{B}$.

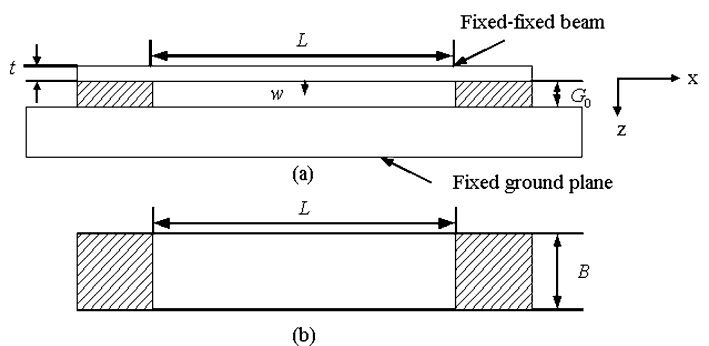

Fig. 7. Detailed schematic of fixed-fixed bridge structure. a Side view; b Top view

Fig. 6. Schematic of fixed-fixed beam 
(a)

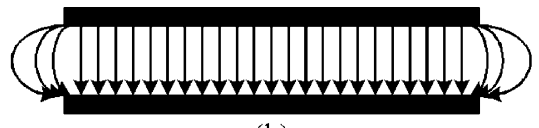

(b)

Fig. 8. Electrostatic field of fixed-fixed bridge structure applied by voltage. a Simplified model; b Schematic including fringing field modification

4.2

\section{Another important factor: axial stress}

The bending of a fixed-fixed beam involves generally a stretching. When the maximum deflection is less than the thickness, small deflection can be considered valid, and the stretching can be neglected. But for RF MEMS switches, the gap $G_{0}$ is usually larger than the beam thickness $t$, so that the maximum deflection at middle point is larger than $t$. Considering the invalidity of small deflection, it is required to take axial stress of the fixed-fixed beam into account.

Axial stress is analyzed in Fig. 9, where $T_{\mathrm{a}}$ is the axial force derived from the elongation of the fixed-fixed beam, and $q$ is the electrostatic force per unit length. is

When a voltage is applied, the actual length of the beam

$L_{2}(x)=\int_{0}^{L}\left[1+\left(\frac{\mathrm{d} w}{\mathrm{~d} x}\right)^{2}\right]^{1 / 2} \mathrm{~d} x$

Considering $L \gg w$, hence $(\mathrm{d} w / \mathrm{d} x)^{2} \ll 1$, as a result, the elongation is approximately given by

$\Delta L \approx \frac{1}{2} \int_{0}^{L}\left(\frac{\mathrm{d} w}{\mathrm{~d} x}\right)^{2} \mathrm{~d} x$

Therefore, axial force is calculated as

$T_{\mathrm{a}}=\sigma B t \approx \tilde{E} B t(\Delta L / L)=\frac{\tilde{E} B t}{2 L} \int_{0}^{L}\left(\frac{\mathrm{d} w}{\mathrm{~d} x}\right)^{2} \mathrm{~d} x$.

\section{3}

\section{Governing equation}

Considering factors as mentioned in Sect. 4.1 and 4.2, the governing equation can be written as

$\tilde{E} \widehat{I} \frac{\partial^{4} w}{\partial x^{4}}-\left(T_{\mathrm{a}}+T_{\mathrm{r}}\right) \frac{\partial^{2} w}{\partial x^{2}}=\frac{\varepsilon_{0} V^{2} B}{2\left(G_{0}-w\right)^{2}}\left(1+f_{\mathrm{f}}\right)$.

Substituting $T_{\mathrm{a}}, T_{\mathrm{r}}, f_{\mathrm{f}}$ and $\widehat{I}=B t^{3} / 12$ into Eq. (10), the governing equation can be expressed as

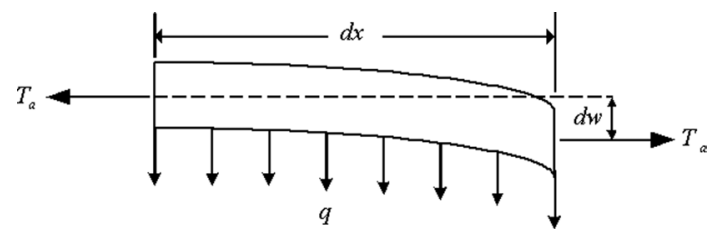

Fig. 9. Analytical schematic of the element of fixed-fixed beam

$$
\begin{gathered}
\frac{\tilde{E} t^{3}}{12} \frac{\partial^{4} w}{\partial x^{4}}-\left[\frac{\tilde{E} t}{2 L} \int_{0}^{L}\left(\frac{\mathrm{d} w}{\mathrm{~d} x}\right)^{2} \mathrm{~d} x+\hat{\sigma} t\right] \frac{\partial^{2} w}{\partial x^{2}} \\
=\frac{\varepsilon_{0} V^{2}}{2\left(G_{0}-w\right)^{2}}\left(1+0.65 \frac{G_{0}-w}{B}\right) .
\end{gathered}
$$

5

Dimensionless numbers for the electromechanical model The behavior of a fixed-fixed beam involves more physical quantities in present model compared with the classical ones. In this section, it will be shown that the dimensionless numbers can play an important role in the present problem.

By making Eq. (11) dimensionless, dimensionless numbers with evident physical significations can be obtained. Introducing the dimensionless transformation as follows

$X=\frac{x}{L}, \quad W=\frac{w}{G_{0}}$,

$\partial^{n} w / \partial x^{n}$ can be shown as

$\frac{\partial^{n} w}{\partial x^{n}}=\frac{G_{0}}{L^{n}} \frac{\partial^{n} W}{\partial X^{n}} \quad(n=1,2,3 \cdots \cdots)$.

Substituting Eqs. (12), (13) into Eq. (11), the governing equation can be expressed as the dimensionless form

$$
\begin{gathered}
\frac{\tilde{E} t^{3} G_{0}}{12 L^{4}} \frac{\partial^{4} W}{\partial X^{4}}-\left[\frac{\tilde{E} t G_{0}^{3}}{2 L^{4}} \int_{0}^{1}\left(\frac{\mathrm{d} W}{\mathrm{~d} X}\right)^{2} \mathrm{~d} X+\frac{\hat{\sigma} t G_{0}}{L^{2}}\right] \frac{\partial^{2} W}{\partial X^{2}} \\
=\frac{\varepsilon_{0} V^{2}}{2 G_{0}^{2}}\left[\frac{1}{(1-W)^{2}}+0.65 \frac{G_{0}}{B} \frac{1}{(1-W)}\right] .
\end{gathered}
$$

Multiplying both sides of Eq. (14) by $2 G_{0}^{2} /\left(\varepsilon_{0} V^{2}\right)$, it becomes

$$
\begin{gathered}
\frac{1}{6} \frac{\tilde{E} t^{3} G_{0}^{3} V^{2} L^{4}}{\frac{\partial^{4} W}{\partial X^{4}}}-\left[\frac{\tilde{E} t G_{0}^{5}}{\varepsilon_{0} V^{2} L^{4}} \int_{0}^{1}\left(\frac{\mathrm{d} W}{\mathrm{~d} X}\right)^{2} \mathrm{~d} X+2 \frac{\hat{\sigma} t G_{0}^{3}}{\varepsilon_{0} V^{2} L^{2}}\right] \frac{\partial^{2} W}{\partial X^{2}} \\
=\left[\frac{1}{(1-W)^{2}}+0.65 \frac{G_{0}}{B} \frac{1}{(1-W)}\right]
\end{gathered}
$$

Several dimensionless numbers are derived as follows

$$
\begin{array}{ll}
\Pi_{1}=\frac{\tilde{E} t^{3} G_{0}^{3}}{\varepsilon_{0} V^{2} L^{4}}, & \Pi_{2}=\frac{\tilde{E} t G_{0}^{5}}{\varepsilon_{0} V^{2} L^{4}}, \\
\Pi_{3}=\frac{\hat{\sigma} t G_{0}^{3}}{\varepsilon_{0} V^{2} L^{2}}, & \Pi_{4}=\frac{G_{0}}{B}=F_{\mathrm{f}},
\end{array}
$$

where $\Pi_{1}$ is the ratio between the bending force $\tilde{E} B t^{3} G_{0} / L^{4}$ and the electrostatic force $B \varepsilon_{0} V^{2} / G_{0}^{2}$ per unit length, $\Pi_{2}$ the ratio between the axial force derived from the elongation of the beam $\tilde{E} B t G_{0}^{3} / L^{4}$ and the electrostatic force $B \varepsilon_{0} V^{2} / G_{0}^{2}$ per 
unit length, $\Pi_{3}$ the ratio between the residual force $\hat{\sigma} B t G_{0} / L^{2}$ and the electrostatic force $B \varepsilon_{0} V^{2} / G_{0}^{2}$ per unit length, and $F_{\mathrm{f}}$ the first order fringing-field correction number, equal to the ratio of $G_{0}$ to $B$.

Therefore, Eq. (15) can be rewritten as

$$
\begin{aligned}
\frac{1}{6} & \Pi_{1} \frac{\partial^{4} W}{\partial X^{4}}-\left[\Pi_{2} \int_{0}^{1}\left(\frac{\mathrm{d} W}{\mathrm{~d} X}\right)^{2} \mathrm{~d} X+2 \Pi_{3}\right] \frac{\partial^{2} W}{\partial X^{2}} \\
= & {\left[\frac{1}{(1-W)^{2}}+F_{\mathrm{f}} \frac{0.65}{(1-W)}\right] . }
\end{aligned}
$$

The equation shows that the governing equation of a fixedfixed bridge structure to an applied voltage is solely dependent upon dimensionless number group $\left(\Pi_{1}, \Pi_{2}\right.$, $\left.\Pi_{3}, F_{\mathrm{r}}\right)$.

Considering the ratio of the bending force to the axial force per unit length of the beam as follows

$\frac{\Pi_{1}}{\Pi_{2}}=\left(\frac{t}{G_{0}}\right)^{2}$

we know that when $t$ is more than $G_{0}$, the axial force derived from elongation is negligible compared with the bending force of the beam and can be neglected.

\section{6}

\section{Discussions}

In order to show the validity of these obtained dimensionless numbers in the behavior of electromechanical RF MEMES switch, some other models and some existing solutions of pull-in voltage will be related to these ones in this section.

\section{1}

\section{Some models of RF MEMS switches}

For 2D model of a fixed-fixed bridge structure, the governing equation as shown in Eq. (2) is recast into the dimensionless form as follows

$$
\frac{\Pi_{1}}{6} \frac{\partial^{4} W}{\partial X^{4}}=\frac{1}{(1-W)^{2}} \text {. }
$$

Because of the ignorance of axial stress, residual stress and fringing field effect, the equation is determined by the only dimensionless number $\Pi_{1}$, the ratio between the bending force and electrostatic force per unit length.

If residual stress is taken into account, the governing equation as shown in Eq. (3) can be rewritten as the dimensionless form

$$
\frac{\Pi_{1}}{6} \frac{\partial^{4} W}{\partial X^{4}}-2 \Pi_{3} \frac{\partial^{2} W}{\partial X^{2}}=\frac{1}{(1-W)^{2}} .
$$

If fringing field is also taken into account, the dimensionless form of governing equation as shown in Eq. (4) can be rewritten as

$$
\frac{\Pi_{1}}{6} \frac{\partial^{4} W}{\partial X^{4}}-2 \Pi_{3} \frac{\partial^{2} W}{\partial X^{2}}=\left[\frac{1}{(1-W)^{2}}+F_{\mathrm{f}} \frac{0.65}{(1-W)}\right] \text {. }
$$

If axial stress effect due to large deflection of the fixedfixed bridge structure is also considered, the dimensionless form of the governing equation is turn into the form as shown in Eq. (17).

By comparing Eqs. (17), (19), (20) and (21), we know that the influences of axial stress, residual stress and fringing-field effect are represented by $\Pi_{2}, \Pi_{3}$ and $F_{\mathrm{r}}$, respectively.

\section{2}

\section{Pull-in voltage $\boldsymbol{V}_{\mathrm{pi}}$}

Since the behavior of the switch is determined by at most four dimensionless numbers. As one of important indices of RF MEMS switches, the pull-in voltage $V_{\mathrm{pi}}$ can be represented by these dimensionless numbers.

(i) For the simplest model (1D), considering $K_{\text {eff }}=32 \tilde{E} B t^{3} / L^{3}$ and $A=L B$, the pull-in voltage $V_{\mathrm{pi}}$ in Eq. (1) can be written as

$V_{\mathrm{pi}}=\sqrt{\frac{256}{27} \frac{\tilde{E} G_{0}^{3} t^{3}}{\varepsilon_{0} L^{4}}}$.

Using dimensionless numbers in (16), the equation can be transformed into the following dimensionless form

$\frac{V_{\mathrm{pi}}}{V}=\frac{16}{3 \sqrt{3}} \sqrt{\Pi_{1}}$.

It is easy to see that the dimensionless pull-in voltage is only determined by $\Pi_{1}$, the ratio of the bending force to the electrostatic force per unit length.

(ii) For a fixed-fixed bridge structure, when residual stress and fringing-field effect are taken into account, pullin voltage $V_{\mathrm{pi}}$ obtained in [16] is

$V_{\mathrm{pi}}=\sqrt{\frac{8}{27\left(1+0.42 \frac{G_{0}}{B}\right)}\left(\frac{32 \tilde{E} t^{3} G_{0}^{3}}{\varepsilon_{0} L^{4}}+\frac{8 \hat{\sigma} t G_{0}^{3}}{\varepsilon_{0} L^{2}}\right)}$,

and the equation can be changed into the dimensionless form

$\frac{V_{\mathrm{pi}}}{V}=\frac{8}{3} \sqrt{\frac{4 \Pi_{1}+\Pi_{3}}{3\left(1+0.42 F_{\mathrm{r}}\right)}}$.

It is evident that the dimensionless pull-in voltage depends on fringing-field effect number, the ratio of the bending force to the electrostatic force per unit length, and the ratio of the axial force to the electrostatic force per unit length.

(iii) For a fixed-fixed bridge structure, if ignoring axial stress and fringing-field effect, an analytic solution for $V_{\mathrm{pi}}$ can be obtained as follows, using the Rayleigh-Ritz method $[14,15]$

$V_{\mathrm{pi}}=\frac{\pi^{2}}{L^{2}} \sqrt{\frac{4 \sqrt{3 / 5} \tilde{E} t^{3} G_{0}^{3}}{25 \varepsilon_{0}}\left(1+\frac{3 \hat{\sigma} L^{2}}{\pi^{2} \tilde{E} t^{2}}\right)}$.

By using the dimensionless numbers in (16), the equation can be rewritten as dimensionless form

$\frac{V_{\mathrm{pi}}}{V}=\frac{2 \pi^{2}}{5} \sqrt[4]{\frac{3}{5}} \sqrt{\left(\Pi_{1}+\frac{3}{\pi^{2}} \Pi_{3}\right)}$. 


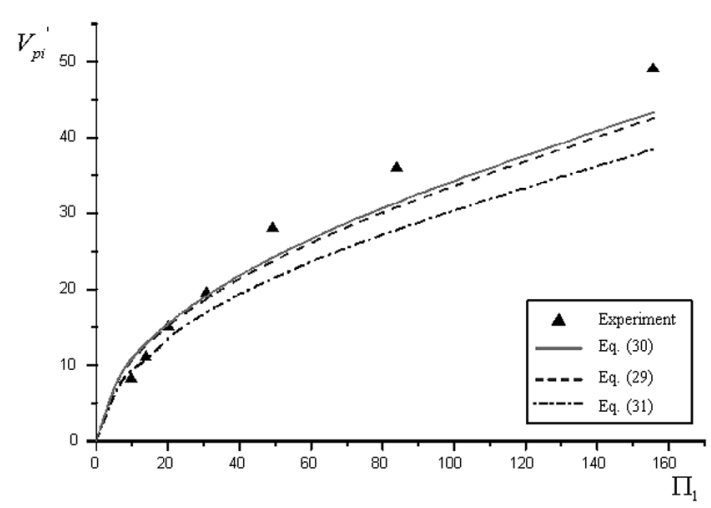

Fig. 10. Use of $\Pi_{1}$ to compare the experimental results with simple theoretical models of fixed-fixed bridge structures $\boldsymbol{\Delta}$ experimental results due to variant length and thickness of the beam; - , - - -, .-.-.- theoretical analyses due to different models

It is evident that the dimensionless pull-in voltage depends upon the ratios of the bending force and residual force to electrostatic force per unit length.

(iv) Solution of pull-in voltage involving the fringing field effect is obtained [15] as follows

$V_{\mathrm{pi}}=\sqrt{\frac{11.86 \tilde{E} t^{3} G_{0}^{3}}{\left(1+0.42 \frac{G_{0}}{B}\right) \varepsilon_{0} L^{4}}}$,

which can be rewritten into the following dimensionless form

$\frac{V_{\mathrm{pi}}}{V}=\sqrt{\frac{11.86}{1+0.42 F_{\mathrm{r}}} \Pi_{1}}$.

Ignoring the factors of residual stress, fringing field effect and axial stress, Eq. (27) can be rewritten as

$\frac{V_{\mathrm{pi}}}{V}=\frac{2 \pi^{2}}{5} \sqrt[4]{\frac{3}{5}} \sqrt{\Pi_{1}}$,

and Eq. (25) can be recast as the following form

$\frac{V_{\mathrm{pi}}}{V}=\frac{16}{3 \sqrt{3}} \sqrt{\Pi_{1}}$,

which is same to Eq. (23).

In order to show its validity to predict the pull-in voltage of switches, Fig. 10 illustrates the use of $\Pi_{1}$ for comparison of the experimental results [15] with simple theoretical models of fixed-fixed bridge structures ignoring the residual stress, the axial stress and the fringing effect. The horizontal axis represents $\Pi_{1}$, the ratio of the bending force to the electrostatic force per unit length, and the vertical axis represents the dimensionless voltage, i.e., $V_{\mathrm{pi}}^{\prime}$ is $V_{\mathrm{pi}}$ normalized by unit voltage. The solid curve is the theoretical analysis of model based on Eq. (30), similarly the dashed curves are the theoretical analysis of models based on Eq. (29) and Eq. (31), respectively.

When $\Pi_{1}$ is less than about 30 , these theoretical models agree relatively well with the experimental results. However, the difference between prediction of these theoretical models and the experimental results will be larger with increasing $\Pi_{1}$ (depicted in Fig. 10). Obviously, both $\Pi_{2}$ and $\Pi_{3}$ increase with increasing $\Pi_{1}$ due to the changes of parameters $t$ and $L$. In fact, the increment of the difference between the theoretical models and the experimental results is a result of ignorance of axial stress and residual stress. Therefore, to obtain more accurate models, it is necessary to take some these factors into account.

7

\section{Concluding remarks}

Axial stress, residual stress, and fringing-field effect are important in the behaviors of RF switches and can cause degradation or even failure of the devices [22]. An improved fixed-fixed bridge structure model, involving these factors, is established and discussed in this paper. In order to facilitate the analysis of the factors, four dimensionless numbers $\Pi_{1}, \Pi_{2}, \Pi_{3}$ and $F_{\mathrm{f}}$ are presented. $\Pi_{1}$ is the ratio between the bending force and the electrostatic force per unit length, $\Pi_{2}$ the ratio between the axial force and the electrostatic force per unit length, $\Pi_{3}$ the ratio between the residual force and the electrostatic force per unit length, and $F_{\mathrm{f}}$ the fringing-field correction number. Such a simplification not only decreases the enormous and unnecessary repeated work, but also gives a convenient approach of design.

The influences of axial stress, residual stress and fringing-field effect on some obtained models and solutions of pull-in voltage are reformulated and analyzed using dimensionless numbers.

It should be pointed out that dimensional analysis can give only general relationships among these dimensionless numbers. The relative importance of each dimensionless numbers has to be studied further by analytical, experimental and numerical studies.

\section{Appendix}

Table 1. Parameters in the present paper

\begin{tabular}{lll}
\hline Symbol & Physical meaning & Dimension \\
\hline$B$ & Width of beam & $L$ \\
$\tilde{E}$ & Effective modulus of beam & $M L^{-1} T^{-2}$ \\
$f_{\mathrm{f}}$ & $\begin{array}{l}\text { The first order fringing-field } \\
\text { correction }\end{array}$ & Dimensionless \\
$G_{0}$ & Gap between movable and & $L$ \\
& $\quad$ ground plates & \\
$I$ & Moment of the inertia of & $L^{4}$ \\
& $\quad$ cross-section & \\
$K_{\mathrm{eff}}$ & Effective spring constant of beam & $M T^{-2}$ \\
$L$ & Length of beam & $L$ \\
$q$ & Electrostatic force per unit length & $M T^{-2}$ \\
& on beam & \\
$t$ & Thickness of beam & $L$ \\
$T_{\mathrm{a}}$ & Axial force of beam & $M L T^{-2}$ \\
$T_{\mathrm{r}}$ & Residual force of beam & $M L T^{-2}$ \\
$w$ & Deflection of beam & $L$ \\
$V$ & Voltage applied & $M L^{2} T^{-2} Q^{-1}$ \\
$V_{\mathrm{pi}}$ & Pull-in voltage & $M L^{2} T^{-2} Q^{-1}$ \\
$x$ & Longitudinal distance along beam & $L$ \\
$\varepsilon_{0}$ & Vacuum permittivity & $M L^{-1} T^{-3} Q^{2}$ \\
$\hat{\sigma}$ & Residual stress of beam & $M L^{-1} T^{-2}$ \\
\hline
\end{tabular}


Table 2. Dimensionless numbers obtained in the present paper

\begin{tabular}{lll}
\hline Number & Dimensionless number & Comments \\
\hline 1 & $\Pi_{1}=\frac{\tilde{E} t^{3} G_{0}^{3}}{\varepsilon_{0} V^{2} L^{4}}$ & $\begin{array}{l}\text { Ratio between the bending force and the electrostatic } \\
\text { force per unit length. } \\
\text { Ratio between the axial force and the electrostatic } \\
\text { force per unit length. }\end{array}$ \\
3 & $\Pi_{2}=\frac{\tilde{E} t G_{0}^{5}}{\varepsilon_{0} V^{2} L^{4}}$ & $\begin{array}{l}\text { Ratio between the residual force and the electrostatic } \\
\text { force per unit length. }\end{array}$ \\
4 & $\Pi_{3}=\frac{\hat{\sigma} t G_{0}^{3}}{\varepsilon_{0} V^{2} L^{2}}$ & Fringing field correction number. \\
\hline
\end{tabular}

\section{References}

1. Nguyen CT (1998) Microelectromechanical devices for wireless communications. IEEE MEMS'98, Heidelberg, Germany, pp. 1-7

2. Petersen KE (2000) Bringing MEMS to market. Proceedings of Solid-State Sensor and Actuator Workshop, Hilton Head Island, South Carolina, pp. 60-64

3. Tang WC; Lee AP (2001) Defense applications of MEMS. Microelectromechanical Systems. Technology and Applications (MRS Bulletin) 26(4): 318-319

4. Hao YL; Zhang LX; Li T; Zhang DC (2001) The technology of silicon-based MEMS. Journal of Mechanical Strength (Special Issue on MEMS) 23(4): 523-526 (in Chinese)

5. Feng ZP; Zhang HT; Zhang WG; Su BZ; Gupta KC; Bright VM; Lee YC (2000) MEMS-based variable capacitor for millimeter-wave applications. Proceedings of Solid-State Sensor and Actuator Workshop, Hilton Head Island, South Carolina, pp. $255-258$

6. Elders J; Spiering V; Walsh S (2001) Microsystems Technology (MST) and MEMS applications: an overview. Microelectromechanical Systems: Technology and Applications (MRS Bulletin) 26(4): 312-317

7. Schiele I; Huber J; Hillerich B; Kozlowski F (1998) Surfacemicromachined electrostatic microrelay. Sensors and Actuators A (66): 345-354

8. Legtenberg R; Gilbert J; Senturia SD (1997) Electrostatic curved electrode actuators. J Microelectromech Syst 6(3): 257-265

9. Zhao YP (1998) Suggestion of a new dimensionless number for dynamic plastic response of beams and plates. Archive of Applied Mechanics 68(7/8): 524-538

10. Li QM; Jones $\mathbf{N}(2000)$ On dimensionless numbers for dynamic plastic response of structural members. Archive of Applied Mechanics 70: 245-254

11. Park JY; Kim GH; Chung KW; Bu JU (2001) Monolithically integrated micromachined RF MEMS capacitive switches. Sensors and Actuators A (89): 88-94

12. Shi F; Ramesh P; Mukherjee S (1995) Simulation methods for Micro-Electro-Mechanical Structures (MEMS) with application to a microtweezer. Computers and Structures 56(5): 769-783

13. Yao JJ (2000) Topical review: RF MEMS from a device perspective. J Micromech Microeng 10: R9-R38

14. Osterberg PM; Senturia SD (1997) M-test: a test chip for MEMS material property measurement using electrostatically actuated test structures. J Microelectromech Syst 6(2): 107118

15. Gupta RK (1997) Electrostatic pull-in test structure design for in-situ mechanical property measurements of MicroElectro-Mechanical Systems (MEMS). Ph.D. Thesis, the Massachusetts Institute of Technology, USA

16. Huang JM; Liew KM; Wong CH; Rajendran S; Tan MJ; Liu AQ (2001) Mechanical design and optimization of capacitive micromachined switch. Sensors and Actuators A (93): 273285

17. Chan EK; Garikipati K; Dutton RW (1999) Characterization of contact electromechanics through capacitance-voltage measurements and simulations. J Microelectromech Syst 8(2): 208-217

18. Osterberg PM; Yie H; Cai X; White J; Senturia SD (1994) Selfconsistent simulation and modeling of electrostatically deformed diaphragms. IEEE MEMS'94, Oiso, Japan, pp. 28-32

19. Gretillat MA; Gretillat F; Rooij NF (1999) Micromechanical relay with electrostatic actuation and metallic contacts. J Micromech Microeng 9: 324-331

20. Senturia SD (1987) Microfabricated structures for the measurement of mechanical properties and adhesion of thin films. Proc Transducers'87, Tokyo, Japan, pp. 11-16

21. Qian J; Liu C; Zhang DC; Zhao YP (2001) Residual stresses in Micro-Electro-Mechanical Systems. Journal of Mechanical Strength (Special Issue on MEMS) 23(4): 393-401 (in Chinese)

22. Zhang LX; Zhang JW; Zhao YP; Yu TX (2002) Failure modes of doubly supported capacitive RF MEMS switches. International Journal of Nonlinear Sciences and Numerical Simulation 3(3/ 4): $353-356$ 